\title{
Study of prevalence and antimicrobial susceptibility of blood culture bacterial isolates
}

\author{
Ehwarieme Daniel Ayobola*, Egbule,Olivia Sochi and Omonigho,Ovuokeroye \\ Department of Microbiology, Delta State University Abraka Nigeria. \\ E-mail: ayoboladaniel@yahoo.com
}

Received 25 July 2010; received in revised form 10 October 2010; accepted 6 November 2010

\begin{abstract}
Bloodstream infections are associated with significant morbidity and mortality. Definitive diagnosis is by bacteriologic culture of blood samples to identify organisms and establish antibiotic susceptibility. Between July and September 2009 , 249 blood samples collected from patients at the University of Benin Teaching Hospital were processed. Positive cultures which accounted for $48(19.3 \%)$ of total samples screened, were purified and identified according to standard methods. Sensitivity of bacteria to different antibiotics was determined by Kirby-Bauer disk diffusion method. Microorganisms recovered were Staphylococcus aureus (14.6\%), Providencia spp., Pseudomonas aeruginosa, Enterobacter spp., Klebsiella pneumoniae and Proteus mirabilis (12.5\% respectively), Escherichia coli and Staphylococcus epidermidis (8.3\% respectively) and Citrobacter freundii (6.3\%). The highest antibiotic activities against Gram positive isolates were observed for ofloxacin $(90.9 \%)$, nitrofurantoin $(81.8 \%)$ and gentamicin $(72.7 \%)$, while in Gram negative bacteria, ofloxacin $(81.1 \%)$ and nalidixic acid $(45.9 \%)$ were most effective. The possibility of drug resistance acquisition by bacteria makes continuous surveillance of antimicrobial susceptibility patterns of bacteria essential as this will enhance efforts to identify resistance and attempt to limit its spread.
\end{abstract}

Keywords: bloodstream infections, blood culture, antibiotic susceptibility

\section{INTRODUCTION}

Bacteremia, the presence of bacteria in the blood, and other Bloodstream Infections (BSIs) are a major cause of morbidity and mortality worldwide (Diekema et al., 2003). Usually, the bloodstream is sterile. Individuals with bacteremia may develop Septicemia, a life-threatening condition in which multiplying bacteria release toxins into the bloodstream and trigger the release of cytokines, causing fever, chills, malaise and lethargy, with difficulty in breathing especially in children (Asindi and Ekanem,1988; Asindi et al.,1991). In the United States alone, about 2 million patients per year, acquire infections in the hospital and, approximately $10-20 \%$ of these are BSIs, with another $4-5 \%$ of these BSIs being fatal (Wenzel and Edmond, 2001). Respiratory, genitourinary tract and intraabdominal foci are identifiable sources of BSIs (Jarvis, 2002).

Rapid immunological techniques like C-Reactive Protein (CRP) assay may serve as a rapid screening procedure to detect a BSI so as to commence treatment but, the test is unspecific and fails to give an insight into the particular pathogen involved. Hence, blood culture remains the highly specific indicator of bacteremia and, antimicrobial susceptibility test assist a great deal in precise identification of the most appropriate choice of drug to be administered (Bohte et al., 1995). However, blood culture techniques take a minimum of 7 days for definitive results- a time span that may not be available for the desperately sick patient. This is because, early administration of drugs to patients with BSI drastically reduce cases of mortality (Diekema et al., 1999).

It is therefore necessary to periodically document research results obtained from analysis of blood culture, to assist clinicians have the needed idea to commence treatment for desperately sick patients, awaiting blood culture results.

This study reports the prevalence, as well as antibiotic resistance profile of isolates obtained from the blood of adults and children/neonates attending the University of Benin Teaching Hospital, a tertiary health care facility serving a great part of the Western Niger Delta.

\section{MATERIALS AND METHODS}

\section{Sample collection}

A total of two hundred and forty nine blood samples were collected from different wards in the University of Benin teaching Hospital (UBTH) from patients of various ages, between July 2009 and September 2009.

Blood was aseptically collected from patients (about $10 \mathrm{~mL}$ of blood from an adult and $2 \mathrm{~mL}$, from a young child) and equally dispensed into 2 culture bottles, one 
containing thioglycollate broth and the other, Brain heart infusion broth. Culture bottles were clearly labeled with the name, number of the patient and the date and time of collection. Culture bottles were then incubated at $37^{\circ} \mathrm{C}$ for up to 7 days or for a maximum of 14 days anaerobically and aerobically. Bottles were macroscopically examined daily for visible evidence of bacteria growth such as turbidity, haemolysis, gas bubbles and clots. For positive blood samples, subcultures were made onto blood agar, chocolate agar and MacConkey agar (HI-MEDIA, Mumbai, India). The media were similarly incubated. Characteristic colonies produced were examined and identified morphologically and biochemically as per standard procedures (Cheesbrough, 2000).

\section{Antimicrobial Susceptibility Test}

The antimicrobial susceptibility test was performed for isolated organisms by Kirby-Bauer's disk diffusion technique according to the Clinical and Laboratory Standards Institute (CLSI, 2002) guideline. Antibiotic disks (Abtek Biologicals Ltd) and their concentrations per disk $(\mu \mathrm{g})$ comprised: amoxicillin (25), cotrimoxazole (25), nitrofurantoin (300), gentamicin (10), nalidixic acid (30), ofloxacin (20), augmentin (amoxicillin-clavulanate) (30) and tetracycline (30).

A sterile wire loop was used to pick a well isolated colony of test organism into $4 \mathrm{~mL}$ of sterile physiological saline. This was incubated for $2 \mathrm{~h}$ at $37^{\circ} \mathrm{C}$ and the turbidity of the suspension was matched with a $0.5 \%$ McFarland standard.

A sterile cotton swab stick was dipped into the broth, pressed against the sides of the bottle wall to reduce the amount of fluid and used to streak the surface of sensitivity agar (HI-MEDIA). The surface of the agar was allowed to dry and then the sensitivity disc was placed on the surface of the agar with the aid of sterile forceps. Plates were left to stand for 15 min to allow for absorption of the disc into the agar and then incubation followed at 37 ${ }^{\circ} \mathrm{C}$ for $24 \mathrm{~h}$.

Zones of inhibition obtained were measured and compared against a standard interpretative chart (Cheesbrough, 2000).

\section{RESULTS}

From total blood culture screened during the study period, $48(19.3 \%)$ were positive for bacterial growth. These belonged to 9 adults (18.8\%), 11 children (22.9\%) and 28 $(58.3 \%)$ neonates. The microorganisms which were recovered from blood cultures when gram stained include: $37(77.1 \%)$ Gram negative bacilli and 11 (22.9\%) Gram positive cocci. The most commonly isolated organism from all patients was Staphylococcus aureus 7 (14.6\%), followed by an equal frequency of occurrence of Providencia spp., Pseudomonas aeruginosa, Enterobacter spp. Klebsiella pneumoniae and Proteus mirabilis; each having $6(12.5 \%)$ isolates. The least common were Citrobacter freundii 3 (6.3\%), Escherichia coli 4 (8.3\%) and Staphylococcus epidermidis $4(8.3 \%)$ as represented in Figure 1.

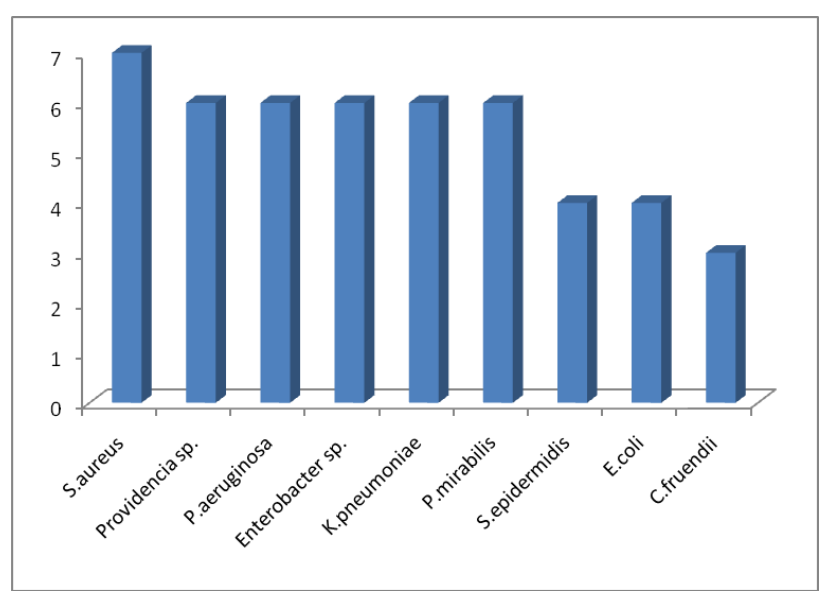

Figure 1: Frequency of bacteria isolated from blood of patients. $x$-axis: blood isolates ; $y$-axis: number of isolates.

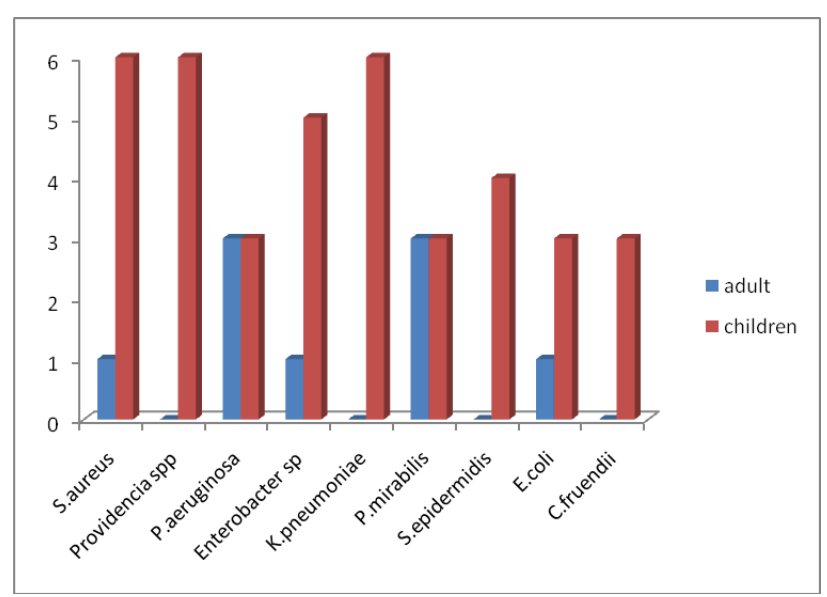

Figure 2: Comparative frequency of bacteria isolated from blood culture obtained from adults vs children/neonates. $x$-axis: blood isolates; $y$-axis: number of isolates.

Amongst the positive cultures obtained from adults, the most commonly isolated organisms were Pseudomonas aeruginosa and Proteus mirabilis, each with an equal frequency of $3(33.3 \%)$. E. coli, S. aureus, and Enterobacter spp., each have 1(11.1\%) while Providenia sp., K. pneumoniae, $S$. epidermidis and $C$. fruendii were not isolated from adults, as represented in Figure 2. 
Table 1: Antibiotic resistance pattern of bacteria isolated from blood culture (\% resistance).

\begin{tabular}{|c|c|c|c|c|c|c|c|c|}
\hline Isolate & OFL & NIT & NAL & COT & GEN & TET & $\mathrm{AUG}$ & AMX \\
\hline $\begin{array}{l}P . \text { aeruginosa. } \\
(n=6)\end{array}$ & $2(33.3 \%)$ & $5(83.3 \%)$ & $3(50 \%)$ & $5(83.3 \%)$ & $4(66.7 \%)$ & $6(100 \%)$ & $6(100 \%)$ & $5(83.3 \%)$ \\
\hline $\begin{array}{l}\text { Providencia spp. } \\
(\mathrm{n}=6)\end{array}$ & $0(0.00 \%)$ & $5(83.3 \%)$ & $3(50 \%)$ & $5(83.3 \%)$ & $5(83.3 \%)$ & $6(100 \%)$ & $6(100 \%)$ & $6(100 \%)$ \\
\hline $\begin{array}{l}\text { Citrobacter } \\
\text { feundii } \\
(\mathrm{n}=3)\end{array}$ & $0(0.00 \%)$ & $0(0.00 \%)$ & $0(0.00 \%)$ & $1(33.3 \%)$ & $2(66.7 \%)$ & $3(100 \%)$ & $3(100 \%)$ & $3(100 \%)$ \\
\hline $\begin{array}{l}\text { S. aureus } \\
(\mathrm{n}=7)\end{array}$ & $0(0.00)$ & $0.00 \%)$ & $4(57.1 \%)$ & $3(42.9 \%)($ & $2(28.6 \%)$ & $5(71.4 \%)$ & $5(71.4 \%)$ & $6(85.7 \%)$ \\
\hline $\begin{array}{l}\text { P. mirabilis } \\
(\mathrm{n}=6)\end{array}$ & $3(50 \%)$ & $5(83.3 \%)$ & $3(50 \%)$ & $6(100 \%)$ & $5(83.3 \%)$ & $6(100 \%)$ & $6(100 \%)$ & $6(100 \%)$ \\
\hline $\begin{array}{l}\text { S. epidermidis } \\
(n=4)\end{array}$ & $1(25 \%)$ & $2(50 \%)$ & $2(50 \%)$ & $3(75 \%)$ & $1(25 \%)$ & $4(100 \%)$ & $3(75 \%)$ & $2(50 \%)$ \\
\hline $\begin{array}{l}\text { Enterbacter } \\
\text { spp } .(n=6)\end{array}$ & $0(0.00 \%)$ & $6(100 \%)$ & $5(83.3 \%)$ & $6(100 \%)$ & $5(83.3 \%)$ & $6(100 \%)$ & $6(100 \%)$ & $6(100 \%)$ \\
\hline $\begin{array}{l}\text { K. pneumoniae } \\
(\mathrm{n}=6)\end{array}$ & $0.00 \%)$ & $5(83.3 \%)$ & $4(66.7 \%)$ & $5(83.3 \%)$ & $5(83.3 \%)$ & $6(100 \%)$ & $6(100 \%)$ & $6(100 \%)$ \\
\hline $\begin{array}{l}\text { E. coli } \\
(\mathrm{n}=4)\end{array}$ & $2(50 \%)$ & $4(100 \%)$ & $2(50 \%)$ & $2(50 \%)$ & $2(50 \%)$ & $4(100 \%)$ & $4(100 \%)$ & $4(100 \%)$ \\
\hline
\end{tabular}

OFL: Ofloxacin $(20 \mu \mathrm{g})$; NIT: Nitrofurantoin $(300 \mu \mathrm{g})$; NAL: Nalidixic acid $(30 \mu \mathrm{g})$; COT: Cotrimoxazole $(25 \mu \mathrm{g})$; GEN: Gentamicin (10 $\mu \mathrm{g})$; TET: Tetracycline $(30 \mu \mathrm{g})$; AUG: Augmentin $(30 \mu \mathrm{g})$; AMX: Amoxicillin $(25 \mu \mathrm{g})$; $\mathrm{n}$ : Total number of isolates

The positive cultures obtained from children/neonates indicated that Klebsiella pneumoniae and Providencia spp. had the highest number of isolates, 6 (15.4\%) while the least number of isolates obtained was recorded for $E$. coli, $C$. freundii, $P$. aeruginosa, and $P$. mirabilis all with 3 $(7.7 \%)$ respectively (Figure 2 ). As could be observed, the general prevalence of bacterial isolates was higher in children/neonates, as compared with adults.

From the results of antibiotic susceptibility testing, all the bacteria recovered from blood culture showed the highest degree of resistance to tetracycline (95.8\%), augmentin (93.8\%) and amoxicilin (91.7\%).The Gram negative bacilli showed a high degree of resistance to commonly used antibiotics with the highest to tetracycline and augmentin (100\%), amoxicillin (97.3\%), nitrofurantoin and cotrimoxazole (81.1\%) with a comparatively low resistance to ofloxacin (18.9\%). Gram positive cocci were highly resistant to tetracycline $(81.8 \%)$, augmentin and amoxicillin (72.7\%), but were highly sensitive to ofloxacin (90.9\%) (Table 1).

\section{DISCUSSION}

This study revealed that 48 (19.3\%) out of 249 total samples screened were positive for the presence of bacteria which is in agreement with the study of Arora and Devi, (2007), with a reported frequency of $20.02 \%$. In contrast however, Sobhani et al., (2004), reported a relative frequency of $6.5 \%$. The variation in blood culture positivity is related to different factors such as the amount of blood taken for screening (Lee et al., 2007), the system and type of blood culture medium formulation used for bacterial detection, or even effective antibiotic therapy prior to taking blood for culture (Mehdinejad et al., 2009).

The commonly isolated organisms from this study include: Staphylococcus aureus, Pseudomonas aeruginosa and members of the Enterobacteriaceae. This result is in accordance with the findings obtained by Mehta et al., (2005). They reported that Pseudomonas aeruginosa, Escherichia coli, Klebsiella pneumoniae, Staphylococcus aureus, Salmonella typhi and Enterococcus faecalis were the most frequently isolated organisms. Fluit et al., (2000) and Diekema et al., (2000) reported that $S$. aureus and $E$. coli were the most frequently isolated organisms from hospitalized patients in the United States and Europe. Shehabi and Baadran (1996) reported that species of Staphylococcus, Acinetobacter, Pseudomonas, Enterobacter, Klebsiella 
and Candida were the most prevalent pathogens recovered from Intensive Care Unit (ICU) patients. The prevalence of $S$. epidermidis was not high in this study. This is the major contaminant in blood cultures. This study reported a relative frequency of $8.3 \%$ and this report is similar to that obtained by Mehdinejad et al., (2009) with a reported frequency of $6.45 \%$.

In this study, the most common resistance was seen to tetracycline $(95.8 \%)$, augmentin $(93.8 \%)$ and amoxicillin $(91.7 \%)$ in all isolated bacteria. This result is in accordance with the findings obtained by Edoh and Alomatu, (2007). Their study showed that isolates were highly resistant to tetracycline, ampicillin and cotrimoxazole. Sobhani et al., (2004) also reported that isolates showed highest resistance to amoxicillin. In this study, $S$. aureus isolates were fully sensitive to ofloxacin (100\%), nitrofurantoin (100\%) and showed a high sensitivity to gentamicin $(71.4 \%)$. These findings were in concordance with Mehta et al., (2005). Their isolates showed $89.74 \%$ susceptibility to ciprofloxacin and $61.6 \%$ to gentamicin. For the Gram negative isolates obtained from this study, highest resistance were shown to tetracycline (100\%) and augmentin (100\%) with a comparatively low resistance to ofloxacin (18.9\%). Previous studies by Garg et al., (2007) showed a similar resistance to tetracycline with a percentage resistance of 82.4\%. Mehta et al., (2005) however, recorded a low resistance to augmentin $(55.22 \%)$ as opposed to results from this study. This difference may be due to differences in drug pattern of use by patients. Mehdinejad et al., (2009) reported a percentage resistance of $22.4 \%$ to ciprofloxacin and this result tallied with that from this study.

The increase in the number of isolates resistant to at least 3 drugs is alarming. This phenomenon is termed multidrug resistance. From this study, all the isolates obtained were multi-drug resistant. The problem of antibiotic resistance was recognized and reported in the 1980's where multiple resistant strains were seen (Edoh and Alomatu, 2007). Previous studies by Moniri et al., (2006) showed that 51 out of $69(73.9 \%)$ of their isolates were resistant to three or more antimicrobials. Japoni et al. (2008) recorded the occurrence of multi-drug resistance. Their isolates were resistant to 4 antimicrobial agents. Edoh and Alomatu, (2007) also recorded a resistance to 3 antibiotics by all isolates.

The development of this phenomenon is often facilitated by the increasing use of invasive techniques, immunosuppressive drugs and inappropriate antibiotic therapy (Shehabi and Baadran, 1996). Infections caused by multi-drug resistant bacteria constitutes a serious problem for patients throughout the world (New, 1993;
Verbist, 1993; Emori and Gaynes, 1993; George, 1993). These bacteria are acquired primarily from the hospital. Hence infection control, continuously monitoring the presence of microorganisms and the avoidance of excessive or continued use of any single drug over a period of time would help control the problem of nosocomial infection and drug resistance. Combination therapy would also help in combating the issue of multidrug resistance among BSI isolates. Early initiation of appropriate antimicrobial treatment is critical in decreasing mortality and morbidity among patients with blood stream infections (Diekema et al., 1999). Furthermore, the initiation of such therapy should always be decided based on the knowledge of the likely pathogens and their usual antimicrobial susceptibility pattern (Munson et al., 2003).

\section{REFERENCES}

Arora, U. and Devi, P. (2007). Bacterial profile of blood stream infection and antibiotic resistance pattern of isolates. JK Science Journal of Medical Education and Science 9, 186-190.

Asindi, A. A. and Ekanem, A. D. (1988). Neonatal septicaemia in Calabar, Nigeria. East African Medical Journal 65(5), 335-341.

Asindi, A. A., Ibia, E. O. and Udo, J. J. (1991). Mortality pattern among Nigerian children in the 1980s. Journal of Tropical Medicine and Hygiene 94, 152-155.

Bohte, R., VanFurth, R. and VanDen Broek, P. J.(1995). Aetiology of community acquired pneumonia: a prospective study among adults requiring admission to hospital. Thorax 50, 543-547.

Cheesbrough, M. (2000). District Laboratory Practice in Tropical Countries (Part 2). Low price edition. Cambridge University press, United Kingdom. pp. 434.

Clinical and Laboratory Standards Institute. (2002). Performance standard for antimicrobial susceptibility testing; $12^{\text {th }}$ Informational supplement. M100-S12 CLSI, 22, No.1, Pennsylvania, USA.

Diekema, D. J., Beekmann, S. E., Chapin, K. C., Morel, K. A., Munson, E. and Doern, G. V. (2003). Epidemiology and outcome of nosocomial and community-onset bloodstream infection. Journal of Clinical Microbiology 41, 3655-3660.

Diekema, D. J., Pfaller, M. A., Jones, R. N., Doern, G. V., Kugler, K. C., Beach, M. L., Sader, H. S. and The SENTRY Participants Group. (2000). Trends in antimicrobial susceptibility of bacterial pathogens isolated from patients with 
bloodstream infections in the USA, Canada and Latin America. International Journal Antimicrobial Agents 13, 257-271.

Diekema, D. J., Pfaller, M. A., Jones, R. N., Doern, G. V., Winokur, P. L., Gales, A. C., Sader, H. S., Kugler, K. and Beach, M. (1999). Survey of bloodstream infections due to gram-negative bacilli: frequency of occurrence and antimicrobial susceptibility of isolates collected in the United States, Canada and Latin America for the SENTRY antimicrobial surveillance program, 1997. Clinical Journal of Infectious Diseases 29, 595-607.

Edoh, D. and Alomatu, B. (2007). Comparison of antibiotic resistance patterns between laboratories in Accre East, Ghana. African Journal of Science Technology 8(1), 1-7.

Emori, T. G. and Gaynes, R. P. (1993). An overview of nosocomial infection, including the role of the microbiology laboratory. Clinical Microbiology Reviews 6(4), 428-442.

Fluit, A. C., Jones, M. E., Schmitz, F. J., Acar, J., Gupta, R., Verhoef, J. and the SENTRY Participants Group (2000). Antimicrobial susceptibility and frequency of occurrence of clinical blood isolates in Europe from the SENTRY antimicrobial surveillance program, 1997 and 1998. Clinical Journal of Infectious Diseases 30, 454-460.

Garg, A., Anupurba, S., Garg, J. and Sen, M. R. (2007). Bacteriological profile and antimicrobial resistance of blood culture isolates from a University hospital. Journal of the Indian Academy of Clinical Medicine 8(2), 139-143.

George, D. L. (1993). Epidemiology of nosocomial ventilator-associated pneumonia. Infection Control and Hospital Epidemiology 14(3): 163169.

Japoni, A., Farshad, S., Alborzi, A., Kalani, M., Rafaatpour, N., Oboodi, B. and Pourabbas, B. (2008). Epidemiology and antibacterial susceptibility patterns of bloodstream infections, 2001-2004: An experience with BACTEC 9240 in Southern Iran. Pakistan Journal of Biological Sciences 11(3), 422-427.

Jarvis, W. R. (2002). The evolving world of health careassociated bloodstream infection surveillance and prevention: Is your system as good as you think? Infection Control and Hospital Epidemiology 23, 236-238.

Lee, A., Mirrett, S., Reller, L.B. and Weinstein, M. P. (2007). Detection of bloodstream infections in adults: How many blood cultures are needed. Journal of Clinical Microbiology 45, 3546-3548.

Mehdinejad, M., Khosravi, A. D. and Morvaridi, A. (2009). Study of prevalence and antimicrobial susceptibility pattern of bacteria isolated from blood cultures. Journal of Biological Sciences 9(3), 249-253.

Mehta, M., Dutta, P. and Gupta, V. (2005). Antimicrobial susceptibility pattern of blood isolates from a teaching hospital in North India. Japanese Journal of Infectious Diseases 58, 174-176.

Moniri, R., Mosayebi, A. H., Movahedian, G. H. and Mossavi, A. (2006). Increase of antimicrobial drug resistance in Pseudomonas aeruginosa causing septicaemia. Iranian Journal of Public Health 32, 58-62.

Munson, E. L., Diekema, D. J., Beekmann, S. E., Chapin, K. C. and Doern, G. V. (2003). Detection and treatment of bloodstream infections: Laboratory reporting and antimicrobial management. Journal of. Clinical Microbiology 41, 495-497.

New, H. C. (1993). Infection problems for the 1990's-do we have an answer? Scandinavian Journal of Infectious Diseases 91, 7-13.

Shehabi, A. A. and Baadran, I. (1996). Microbial infection and antibiotic resistance pattern among Jordanian intensive care patients. Eastern Mediterranean Health Journal 2(3), 515-520.

Sobhani, A. Shodjai, H. and Javanbakht, S. (2004). Drug resistance pattern in isolated bacteria from blood cultures. Acta Medica Iranica 42(1), 48-49.

Verbist, L. (1993). Epidemiology and sensitivity of 8625 ICU haematology/oncology bacterial isolates in Europe. Scand. Journal of Infectious Diseases 91, 14-24.

Wenzel, R. P. and Edmond, M. B. (2001). The impact of hospital acquired bloodstream infections. Emerging Infectious Diseases 7, 174-177. 